\title{
Novel Reversible Biotinylated Probe for the Selective Enrichment of Phosphorylated Peptides from Complex Mixtures
}

\author{
Pegah R. Jalili* and Haydn L. Ball \\ Protein Chemistry Technology Center, University of Texas, Southwestern Medical Center, Dallas, Texas, USA
}

To improve the detection of phosphorylated peptides/proteins, we developed a novel protocol that involves the chemical derivatization of phosphate groups with a chemically engineered biotinylated-tag (biotin-tag), possessing three functional domains; a biotin group for binding to avidin, a base-labile 4-carboxy fluorenyl methoxycarbonyl (4-carboxy Fmoc) group, and a nucleophilic sulfhydryl moiety on the side-chain of cysteine. Using this approach, the derivatized, enzymatically digested peptides were selectively separated from unrelated sequences and impurities on immobilized avidin. Unlike previously published phosphopeptide enrichment procedures, this approach upon treatment with mild base liberates a covalently bound Gly-Cys analog of the peptide(s) of interest, exhibiting improved RP-HPLC retention and MS ionization properties compared with the precursor phosphopeptide sequence. The results obtained for a model peptide Akt- 1 and two protein digests, demonstrated that the method is highly specific and allows selective enrichment of phosphorylated peptides at low concentrations of fmol/ $\mu \mathrm{L}$. (J Am Soc Mass Spectrom 2008, 19, 741-750) (C) 2008 American Society for Mass Spectrometry

$P_{n}^{n}$ hosphorylation is an important post-translational modification (PTM) representing a key regulatory mechanism for fundamental cellular events. Routine mapping of this transitory PTM is challenging for the following reasons. First, the negatively-charged phosphate group causes suppression of ionization in positive mode mass spectrometry (MS). Second, low stoichiometry of this modification in complex biological mixtures makes its analysis challenging, and third, their labile nature can affect the ability to assign precise sites of modification. Furthermore, the hydrophilic character of this group may cause some phosphopeptides to elute in the void volume during reversed-phase high-performance liquid chromatography (RP-HPLC) separation [1-4].

These factors underscore the need for continued development of phosphopeptide enrichment methods before mass analysis for identifying phosphorylation sites in a complex biological mixture. Two-dimensional HPLC systems using strong cation-exchange chromatography (SCX), combined with RP-HPLC, as well as immobilized metal affinity chromatography (IMAC) techniques, are widely used for the enrichment of phosphopeptides/proteins [5-14]. In the IMAC approach, the negatively-charged phosphate group on phosphopeptides electroselectively binds to positively charged $\mathrm{Fe}^{3+}$ or $\mathrm{Ga}^{3+}$ ions, which are immobilized on

Address reprint requests to Dr. H. L. Ball, Protein Chemistry Technology Center, UT Southwestern Medical Center, 6001 Forest Park Rd., Dallas, TX, 75390-8816, USA. E-mail: haydn.ball@utsouthwestern.edu

* Current address: Sigma-Aldrich Inc., 2909 Laclede Ave. St. Louis, MO 63103, USA. the stationary phase of the separation column. However, in addition to binding phosphopeptides, these metal ions will also bind nonspecific sequences rich in negatively-charged residues. The nonselective binding of these species can cause the displacement of phosphorylated peptides from the IMAC column resulting in a decrease in the sensitivity of detection for the peptides of interest. Dawoud et al. have combined microfluidic separation techniques with MS for the high throughput, contamination free analysis of phosphoproteins [15]. Recently, some metal oxides such as titanium and zirconium dioxide have been applied to selectively concentrate phosphopeptides from complex biological mixtures [16-19]. Although the titanium dioxide $\left(\mathrm{TiO}_{2}\right)$ enrichment of phosphopeptides has been shown to be very successful, it also suffers from nonspecific binding events. Several groups have attempted to overcome these limitations by either washing the $\mathrm{TiO}_{2}$ column with a solution containing $\mathrm{NH}_{4} \mathrm{Glu}$, which helped to increase recovery by up to $84 \%$ [20]. Wang et al. synthesized a porous anodic alumina membrane for selective enrichment of phosphopeptides [21]. Other groups have used magnetic immobilized metal ions [22] and $\mathrm{Fe}_{3} \mathrm{O}_{4}$ and $\mathrm{ZrO}_{2}$ core-shell micro spheres as affinity probes [23]. However, the labile nature of the phosphate group on phosphopeptides adds an additional element of difficulty with respect to data interpretation, especially in trap-based mass analyzers $[19,24]$. Thus, the $\mathrm{MS}^{3}$ scan mode was developed for ion trap instruments, which has the ability to detect
(C) 2008 American Society for Mass Spectrometry. Published by Elsevier Inc. $1044-0305 / 08 / \$ 32.00$

doi:10.1016/j.jasms.2008.02.004
Published online February 26, 2008 Received November 9, 2007 Revised February 6, 2008 Accepted February 18, 2008 
more diagnostic ions and, as a result, provides more information about phosphorylation sites [24].

Chemical derivatization strategies based on $\beta$ elimination of phosphate moiety of phosphopeptides, followed by a Michael's addition reaction with S- and N-nucleophiles are increasingly being described [25, 26]. Several studies report a combination of chemical derivatization approaches and affinity chromatography for the enrichment of phosphopeptides [27-29]. It was demonstrated that affinity enrichment of phosphopeptides could be achieved by $\beta$-elimination and Michael's addition of biotin or fluorous affinity tags, which were attached covalently but were nonreversible [27, 29]. The former method encountered a problem of incomplete elution of modified biotinylated peptides from the avidin column due to the strong binding affinity between avidin and biotin, resulting in low recovery and loss of phosphopeptide [28].

In the present study, we have attempted to overcome these problems, using a two-step $\beta$-elimination and Michael's addition reaction to replace the phosphate moiety with a reversible biotin-tag (Scheme 1). The dihydroalanine intermediate, which is formed by the loss of the phosphate group in alkaline conditions, reacts with the sulfhydryl group on the side-chain of Cys, resulting in the covalent attachment of the biotintag. Substitution of the phosphate group with the biotin-tag enables the enrichment of phosphopeptides on immobilized avidin. The bound peptides are released into the supernatant upon treatment with a mild base such as triethylamine (TEA), resulting in the addition of Gly-Cys dipeptide at the site of modification whilst leaving biotin-spacer-4-carboxy Fmoc attached to the avidin column. The eluent was then analyzed by MALDI-MS and ESI-MS/MS. The experimental strategy is given in Scheme 2 and represents a refinement of a recently described method in which a reversible His-tag was used to modify and enrich phosphopeptides from complex tryptic protein digests. In that report, selective release from the $\mathrm{Ni}^{2+}$-IMAC column was achieved via a Factor Xa cleavage site incorporated into the peptide sequence between the His-tag and the Gly-Cys dipeptide [29]. The method described here offers some significant advantages in that the tag is smaller and therefore easier to couple, requires mild base treatment instead of expensive Factor Xa to mediate release, and is easier to produce in large quantities. Furthermore, any potential steric problems that may impede access by the enzyme to the Factor Xa cleavage site are also eliminated. We will assess the effectiveness of this novel approach by analyzing phosphopeptides from a variety of sources.

\section{Experimental}

Amino acids and solvents were purchased from Novabiochem (San Diego, CA) or Fisher Scientific (Fair lawn, NJ). Ovalbumin, $\alpha$-casein (bovine) was purchased from SigmaAldrich/New Jersey Center for Biomaterials (Piscataway, NJ). The materials used were barium hydroxide
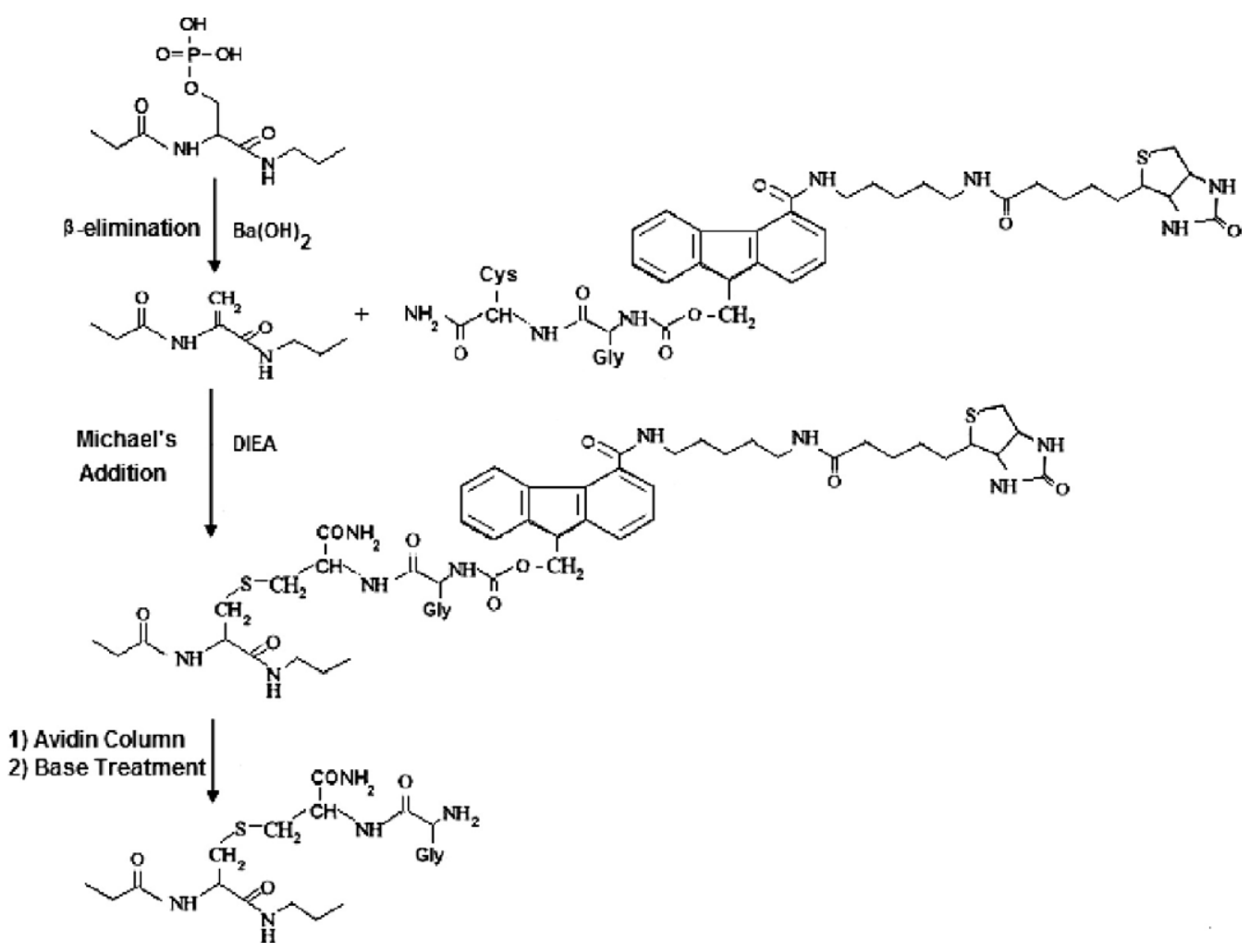

Scheme 1. $\beta$-Elimination and Michael's addition reaction of phosphorylated peptide using biotin-tag. 


\section{Mixture of peptides and phosphorylated peptides}

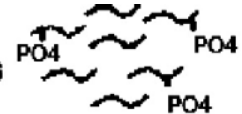

$\downarrow \quad \begin{aligned} & \text { Derivatization } \\ & \text { with Biotin-Tag }\end{aligned}$

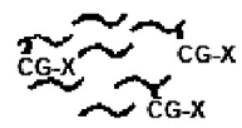

$\downarrow$

IㅔI

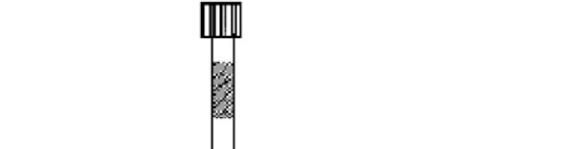

Avidin Column

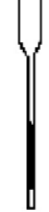

Wash \begin{tabular}{c|} 
Mild Base \\
treatment
\end{tabular}
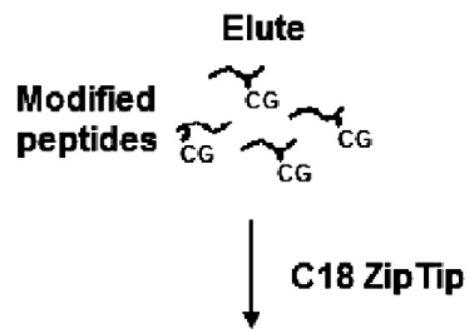

Purify and

Concentrate

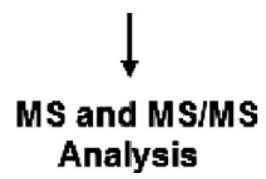

Scheme 2. Biotin enrichment strategy for the selective analysis of phosphorylated peptide.

(Alfa Aesar, Ward Hill, MA), guanidinium $\mathrm{HCl}$, formic acid, trifluoroacetic acid (TFA), hydrogen peroxide, triisopropylsilane (TIS), N,N-diisopropylethylamine (DIEA), and triethylamine (TEA) (Sigma-Aldrich, Saint Louis, MO), immobilized monomeric avidin (Pierce, IL), Poros 20MC (PE Biosystems, Framingham, MA), titanium oxide (ESPI, Ashland, OR), and sequencinggrade modified trypsin, porcine (Promega, Madison, WI); 2,5-dihydroxybenzoic acid (DHB) matrix was purchased from Agilent Technologies (Palo Alto, CA).

\section{Synthesis of Biotinylated Probe (Scheme 3)}

One hundred mg (0.5 mmol) Boc-1,5-diaminopentane was added to $120 \mathrm{mg}$ (0.5 mmol) 4-carboxy Fmoc (1) in $\mathrm{CH}_{2} \mathrm{Cl}_{2}$ :DMF (1:1) in the presence of $105 \mathrm{mg}(0.55 \mathrm{mmol})$ 1-(3-dimethylaminopropyl)-3-ethylcarbodiimide hydrochloride at $0{ }^{\circ} \mathrm{C}$ and stirred for $30 \mathrm{~min}$. The Boc group was removed using $1 \mathrm{~mL}$ of $5 \mathrm{M} \mathrm{HCl}$ in methanol. The mixture was heated and refluxed for $4 \mathrm{~h}$, the solution cooled and then neutralized with $1 \mathrm{M} \mathrm{NaOH}$ in a saturated solution of $\mathrm{NaCl}$. The aqueous solution was extracted with ethyl acetate and the two phases separated. The organic phase, which contains the product (3) was dried over anhydrous $\mathrm{Na}_{2} \mathrm{SO}_{4}$, the solvent removed under reduced pressure to yield 126 $\mathrm{mg}(78 \%)$ of compound (3), which was analyzed by ESI-MS (calc. 324.46; found 324.18). $110 \mathrm{mg}(0.34 \mathrm{mmol})$ compound (3) was added at room temperature to $248 \mathrm{mg}$ $(0.68 \mathrm{mmol})$ of biotin $p$-nitrophenyl ester, stirred overnight, and purified using flash chromatography. The flash chromatography column was prepared by pouring a slurry of silica gel (70-230 mesh, 60A), suspended in dichlormethane:methanol (9:1) running buffer, into a glass chromatography tube $(100 \times 15 \mathrm{~mm})$ with a glass sinter at one end. The compounds were eluted off the column under positive pressure and collected in $2 \mathrm{~mL}$ aliquots. Solvent was removed on a rotary evaporator, resulting in a yield of $124 \mathrm{mg}(67 \%)$ of the desired product (4; calc. 550.79; found 550.26). $120 \mathrm{mg}(0.22 \mathrm{mmol})$ of the biotinylated intermediate (4) was activated with a 2-fold excess of disuccinimidyl carbonate and the product purified on a C18 RP-HPLC semi-preparative column to yield $116 \mathrm{mg}(76 \%)$ of the activated product (5; calc. 691.88; found 691.22). The final biotin-tag (6) was prepared by reacting a 5-fold excess of biotin-spacer 4-carboxy Fmoc succinimidyl ester (5) with Gly-Cys peptidyl-resin for $1 \mathrm{~h}$. The resin was then cleaved for $90 \mathrm{~min}$ using $1 \mathrm{~mL}$ of ( $5: 95 \% \mathrm{vol} / \mathrm{vol}$, triisopropylsilane:TFA) and purified on a C18 RP-HPLC column, with a yield of $25.3 \mathrm{mg}$ of [6] and analyzed by MALDI-MS (Calc. 754.02; found 754.54). Analytical RP-HPLC indicated $95 \%$ purity of the final product (6). Analytical and semi-preparative RP-HPLC was performed on a Waters 717 HPLC system (Waters, Milford, MA), using Vydac C18 analytical $(150 \times 4.6 \mathrm{~mm})$ and semi-preparative $(250 \times 10 \mathrm{~mm})$ columns. Separations were achieved by applying $100 \%$ B linear gradients over $30 \mathrm{~min}$ at $1 \mathrm{~mL} / \mathrm{min}$ or $120 \mathrm{~min}$ at $3 \mathrm{~mL} / \mathrm{min}$, respectively. Solvent A was water $/ 0.045 \% \mathrm{TFA}$ and B acetonitrile $/ 0.036 \%$ TFA. Detection was at $220 \mathrm{~nm}$.

\section{Derivatization}

For the model phosphopeptide; $\beta$-elimination reactions were performed with $10 \mu \mathrm{L}$ of phosphopeptide (10 pmol), $5 \mu \mathrm{L}$ of $8 \mathrm{M}$ guanidine $\mathrm{HCl}, 15 \mu \mathrm{L}$ of acetonitrile, and $10 \mu \mathrm{L}$ of saturated barium hydroxide. After $2 \mathrm{~h}$ reaction time at $45^{\circ} \mathrm{C}$ (final concentration was $250 \mathrm{fmol} / \mu \mathrm{L}$ ), the resulting purified $\beta$-eliminated peptides were desalted on a C18 ZipTip (Millipore, Bed- 
<smiles>O=C(O)c1cccc2c1-c1ccccc1C2CO</smiles>

(1) $\stackrel{\text { (a) }}{\longrightarrow}$<smiles>CC(C)(C)OC(=O)NCCCCNC(=O)c1cccc2c1-c1ccccc1C2CO</smiles>

(2)<smiles>NCCCCCNC(=O)c1cccc2c1-c1ccccc1C2CO</smiles>

(3)

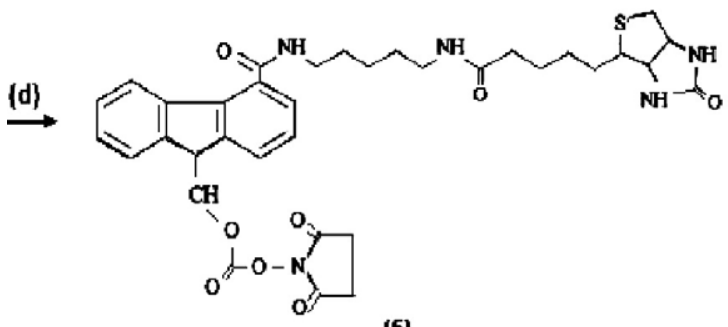

(5)

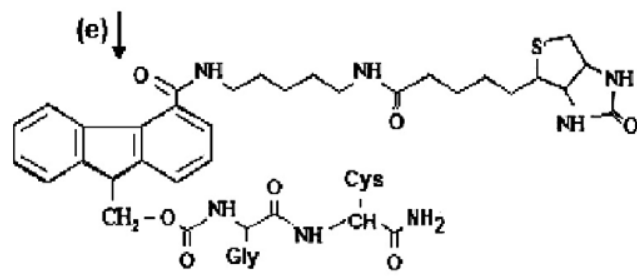

(6)

Scheme 3. Synthesis of the biotin probe. Reagents and conditions: (a) $\mathrm{BtOH}, \mathrm{EDCl}, \mathrm{CH}_{2} \mathrm{Cl}_{2}$, then $\mathrm{H}_{2} \mathrm{~N}\left(\mathrm{CH}_{2}\right)_{5} \mathrm{NHBoc}$. (b) $\mathrm{HCl}$, AcOEt, reflex. (c) Biotin p-nitrophenylester. (d) N,N'-disuccinimidyl carbonate. (e) Gly-Cys resin, reflux, followed by cleavage with 95\%TFA, 5\%TIS.

ford, MA) and then subjected to the Michael's addition reaction. Due to the instability of the urethane bond to $\mathrm{Ba}(\mathrm{OH})_{2}$, the second part of the reaction was performed in $5 \%$ DIEA for $4 \mathrm{~h}$. Thus, $5 \mu \mathrm{L}$ of resulting purified $\beta$-eliminated sample was added to $4 \mu \mathrm{L}$ of purified biotin-tag (6) in ACN:DMF (1:1), $5 \mu \mathrm{L}$ of $8 \mathrm{M}$ guanidine and $1 \mu \mathrm{L}$ of DIEA. The resulting biotinylated peptide was desalted using a C18 ZipTip.

For protein samples; before derivatization, the protein mixture was subjected to performic acid oxidation by adding $50 \mu \mathrm{L}$ of $30 \%$ aqueous hydrogen peroxide: formic acid (5:95 vol/vol) to $20 \mu \mathrm{g}$ of protein. The reaction mixture was incubated overnight at $4{ }^{\circ} \mathrm{C}$ and dried in a centrifugal vacuum system. The dried sample was dissolved in $100 \mu \mathrm{L}$ of $100 \mathrm{mM}$ ammonium bicarbonate. Trypsin digestion of oxidized proteins was performed at $37^{\circ} \mathrm{C}$ overnight using the substrate/ enzyme ratio of 100:1. The resulting mixture was diluted to $1 \mathrm{pmol} / \mu \mathrm{L}$ using $0.1 \%$ formic acid of which 10 $\mu \mathrm{L}$ was used for derivatization using the procedure described above. Final concentration before enrichment was $\sim 250 \mathrm{fmol} / \mu \mathrm{L}$.

\section{$\mathrm{TiO}_{2}$ Enrichment}

$\mathrm{TiO}_{2}$ powder was resuspended in $80 \%$ ACN in $0.1 \%$ TFA and then packed into an Eppendorf gel-loader tip, which was blocked with a glass wool frit. To optimize the binding of protein to the $\mathrm{TiO}_{2}$ microcolumn, the $\mathrm{pH}$ of the digested proteins was adjusted to 6.5. The resulting mixture was introduced into the $\mathrm{TiO}_{2}$ microcolumn and washed with $0.1 \%$ acetic acid, followed by $50 \%$ ACN in $0.1 \%$ acetic acid. The bound phosphopeptides were eluted with $0.5 \%$ ammonium hydroxide ( $\mathrm{pH} 9.5)$.

\section{Avidin Purification}

The biotin-tagged peptides were enriched on a nanoscale avidin column. First, the immobilized avidin resin was equilibrated with the PBS binding buffer ( $0.1 \mathrm{M}$ sodium phosphate and $0.15 \mathrm{M} \mathrm{NaCl}, \mathrm{pH}$ 7.4). A column was prepared by loading the avidin resin into an Eppendorf gel-loader tip and washed with PBS binding buffer. The sample was diluted further in the binding buffer and loaded onto the top of the column. The immobilized 
biotin-tagged peptides were washed with PBS buffer three times and eluted with a solution containing $5 \%$ TEA and $2 \%$ ammonia in water to liberate the Gly-Cys derivatized-peptides (note: biotin-spacer-4-carboxy Fmoc remains attached to the avidin column).

\section{Mass Spectrometry Analysis}

Mass spectrometry analyses were performed using time of flight MALDI-MS (Micromass LR MALDI-TOF; Milford, MA) and QTRAP 4000 triple quadrupole/linear ion trap (MDS SCIEX, Ontario, Canada) instruments. For MALDI-MS analysis, $0.5 \mu \mathrm{L}$ aliquot of a sample was deposited onto a MALDI plate and then $0.5 \mu \mathrm{L}$ of the working matrix 2,5-dihydroxybenzoic acid (DHB) solution slowly applied to the sample droplet. The samplematrix droplet was allowed to air dry at room temperature. Typically, spectra were obtained using a pulsed nitrogen UV laser (337 nm; $3 \mathrm{ns)} \mathrm{in} \mathrm{positive} \mathrm{reflectron}$ mode; pulse voltage of $2800 \mathrm{mV}$; source voltage of 15,000 mV, using the MassLynx 4.0 Software (Waters, Milford, MA). The following settings were used for the QTRAP 4000 experiments: nano-ionspray voltage +2000 $\mathrm{V}$, no heating of the source, curtain gas 20 psi, declustering potential $+110 \mathrm{~V}$. Identification of phosphorylation sites and fragmentation was achieved by using MS-product of Protein Prospector database.

\section{Results and Discussion}

\section{Analytical Strategy}

Published enrichment techniques such as $\mathrm{Fe}^{3+}$ and $\mathrm{Ga}^{3+}$-IMAC, and $\mathrm{TiO}_{2}$, are very reliable ways for isolating phosphopeptides. However they do suffer from some drawbacks, such as copurification of nonspecific, negatively-charged peptides. A convenient, quantitative, site-specific modification of phosphate with a group that exhibits enhanced MS ionization properties is required. We synthesized a biotin tag that was engineered to contain several important features for this purpose. The tag consists of biotin linked to a 4-carboxy Fmoc group through a spacer. The spacer is of sufficient length to reduce steric hindrance between the peptide chain and the biotin molecule, thus optimizing binding to the immobilized avidin. The Fmoc moiety is commonly used in peptide synthesis as an N-terminal protecting group for peptide synthesis. We exploited the base-labile property of this group to provide the desired reversibility, and thus allow the peptides of interest to be released selectively and quantitatively from the avidin column. The Cys residue at one end of the tag provides the necessary sulfhydryl group for reaction with the dihydroalanine intermediate following $\beta$-elimination. Mild base treatment cleaves the urethane bond, leaving the biotin-spacer-4-carboxy Fmoc group attached to the avidin column and releases modified peptide sequence(s) with Gly-Cys covalently attached at the site of phosphorylation. This modifi- cation is more amenable to MS analysis by eliminating the negatively-charged phosphate group, which has a tendency to suppress ionization. The standard $\beta$-elimination/Michael's addition reaction conditions, which are performed at high $\mathrm{pH}$, are not suitable for our probe and therefore it was necessary to modify the two-step procedure. Several bases were tested for use in the Michael's addition step and DIEA proved to have the desired properties in that it did not cleave the Fmoc group and the Michael's addition reaction proceeded well in the presence of the base.

The validity of these assumptions was confirmed with well-defined model phosphopeptides/proteins, using Akt- 1 , ovalbumin, and $\alpha$-casein. The experiments were performed at least three times to optimize the methodology and ensure reproducibility.

\section{Model Peptide (Akt-1)}

A model phosphopeptide Akt-1 was chemically synthesized on an automated peptide synthesizer using Fmoc chemistry with a phosphate group on Ser8 (Figure 1a, calc., 1732.75; found 1732.78). Akt-1 was used to optimize the protocol for the derivatization of phosphate groups with the reversible biotin-tag (6). The MALDI-MS spectrum of $\beta$-eliminated peptide shows the peak of $m / z$ 1634.78 (Figure 1b) for the dihydroalanine intermediate, corresponding to the loss of phosphate. The peak at $\mathrm{m} / \mathrm{z}$ 2387.72 (Figure 1c) represents the phosphopeptide derivatized with biotin-tag. The biotinylated peptide was enriched on the nanoscale avidin column and the immobilized peptide eluted with mild base (5\% TEA and $2 \%$ ammonia in water). The eluent was then acidified with 5\% formic acid and desalted on a C18 ZipTip. The MALDI-MS spectrum of modified peptide after treatment with base, which leaves a Gly-Cys dipeptide at the site of phosphorylation, is shown in Figure 1d. The peak of $m / z 1810.97$ (calc. 1810.82) is clear in the spectrum after enrichment, however, MS/MS sequencing is required to determine the site of phosphorylation unambiguously. Figure 2 shows the MS/MS spectrum of doubly charged ions of Gly-Cys modified peptide at $m / z$ 906.45. The nearly complete series of $b$ and $y$ ions are clear in the resulting MS/MS spectrum that was used to sequence the modified peptide. The data identified the following sequence RPHFPQFS(GC)YSASGTA, thus confirming the site of phosphorylation at Ser8.

The addition of Gly-Cys results in the introduction of an extra protonatable amine group, which enhances the ionization efficiency of the peptide in positive-mode MS, compared with precursor phosphorylated peptide. Furthermore, the labile nature of phosphate group complicates data interpretation requiring the need to run $\mathrm{MS}^{3}$ experiments for complete sequence information. Our approach offers some distinct advantages because there is no loss of the covalently attached Gly-Cys dipeptide in the mass spectrometer. 


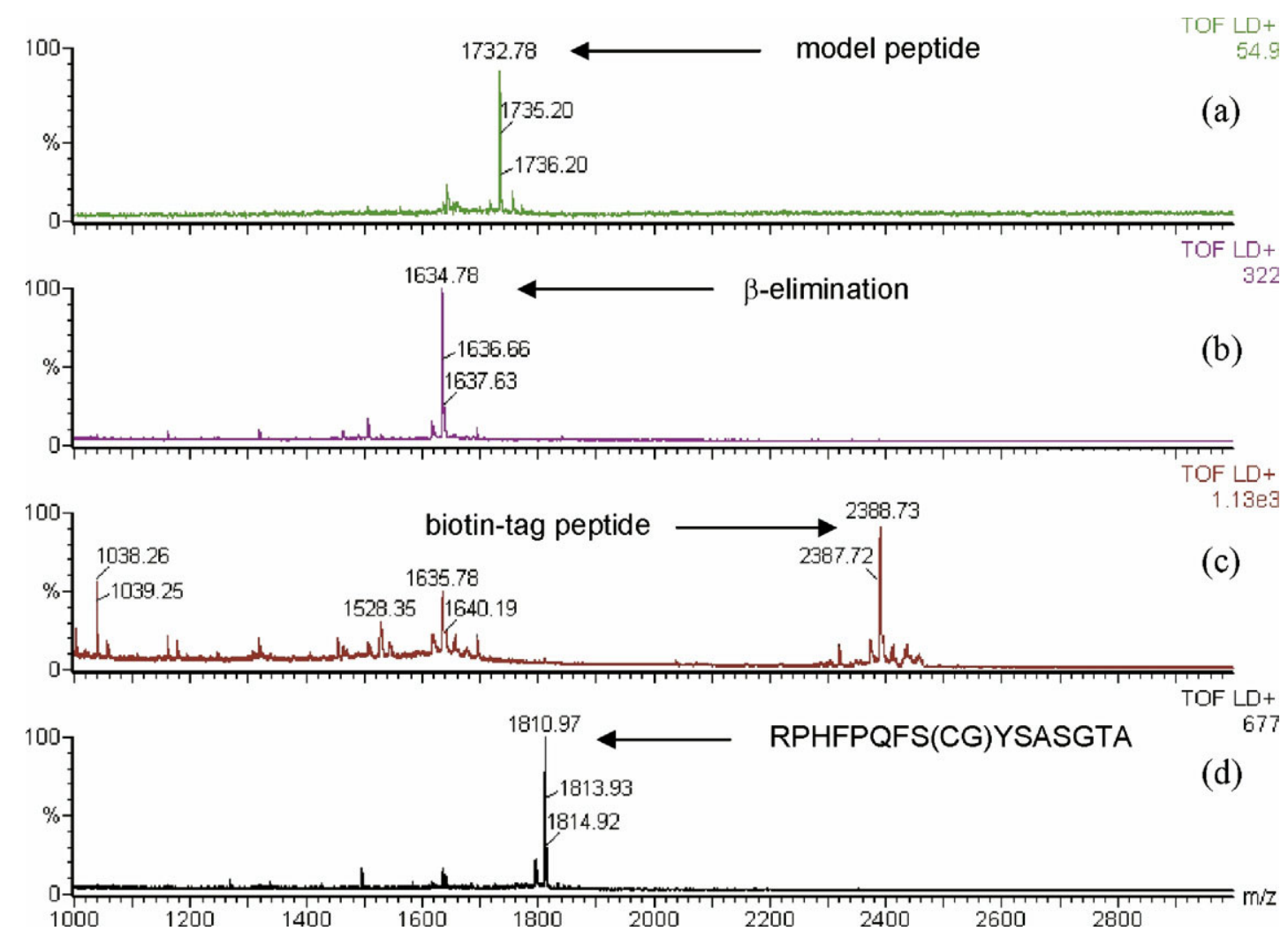

Figure 1. The MALDI-MS spectra of (a) model phosphorylated peptide, after (b) $\beta$-elimination, (c) Michael's addition, and (d) avidin purification and base treatment. The modified peptide has Gly-Cys at the phosphorylation site after avidin purification and base treatment.

Tryptic Digest; Ovalbumin

To evaluate this methodology further we applied the biotin-tag to the chemical derivatization of ovalbumin, which possesses two sites of phosphorylation. Before chemical derivatization, the protein was subjected to performic acid treatment, which oxidizes cysteine, me-
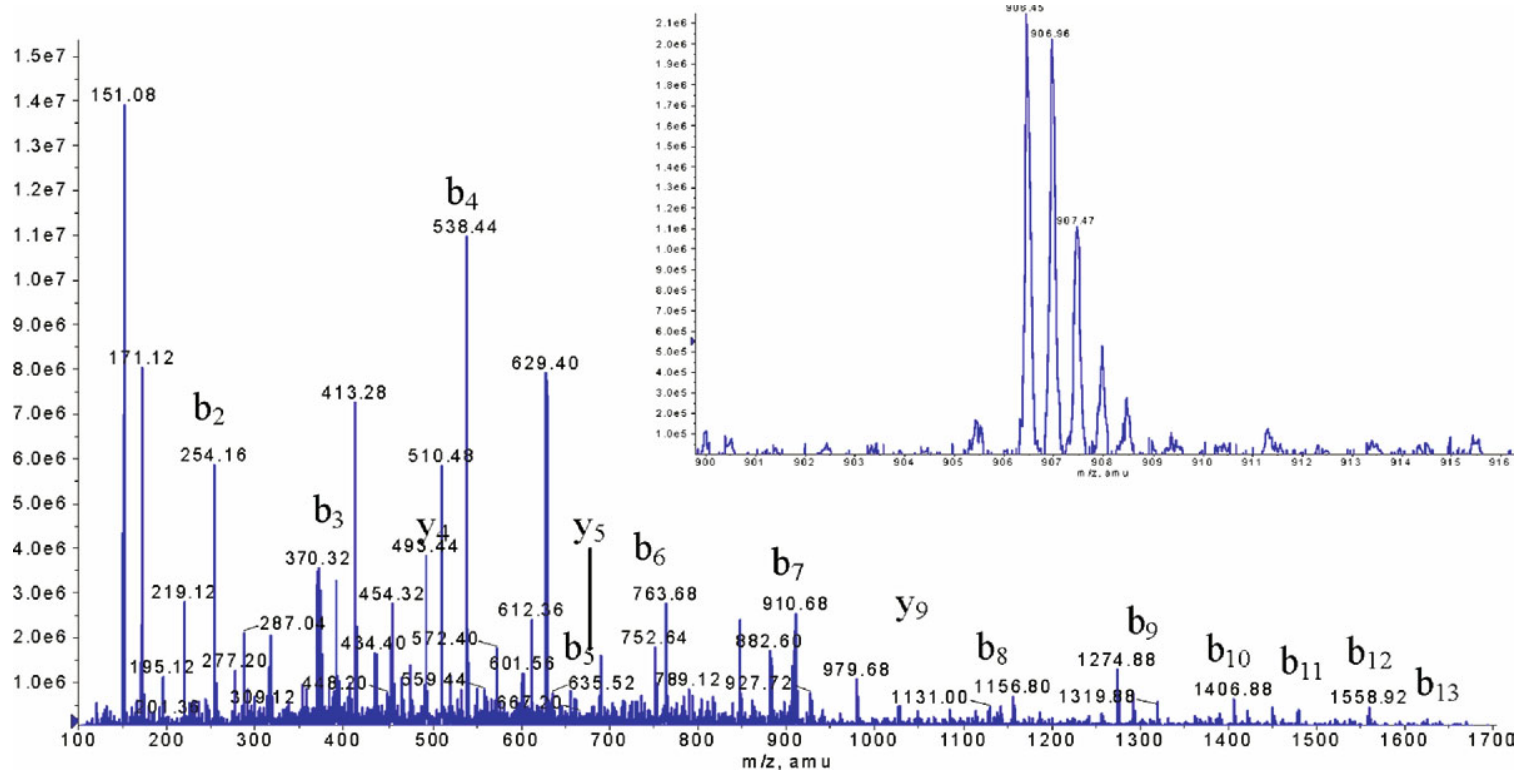

\begin{tabular}{r|r|}
$\mathrm{b}_{3}$ & $\mathrm{y}_{4}$ \\
370.32 & $4_{49}$
\end{tabular} $\mathrm{y}_{5} \quad \mathrm{~b}_{6}$

$\mathrm{b}_{7}$

$763.68-\mathrm{b}_{7}$

$\begin{array}{ll}910.68 & y_{9}\end{array}$

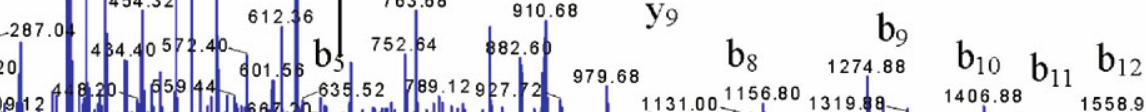

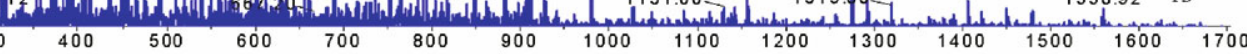

Figure 2. (Insert) MS/MS spectrum of $m / z$ 906.45. The corresponding sequence is RPHFPQFS(GC)YSASGTA. The enhanced resolution MS spectrum of doubly charged Gly-Cys modified peptide at $\mathrm{m} / \mathrm{z}$ 906.45 after avidin purification and base treatment. 
thionine, and tryptophan residues. This pretreatment blocks any undesirable side reactions that may occur between the Cys residue on the biotin-tag and Cys residues present in the ovalbumin sequence. The MALDI-MS spectrum of tryptic digest of ovalbumin is shown in Figure 3a. Two arrows in this figure indicate the peaks corresponding to phosphorylated peptides. The peak intensity of the phosphopeptides is low compared with the other tryptic peptides present in the sample. For comparison of our novel method and IMAC, the phosphorylated peptides in the tryptic mixture were also enriched using $\mathrm{TiO}_{2}$. The peaks of phosphorylated peptides at $m / z 2088.82$ and 2893.21, corresponding to EVVGSAEAGVDAASVSEEFR and FDKLPGFGDSIEAQCGTSVNVHSSLR, respectively, gain significant intensity upon $\mathrm{TiO}_{2}$ enrichment (Figure 3b). However, the peaks of nonspecific peptides are also present, resulting in a more complex spectrum. Figure $3 \mathrm{c}$ shows phosphopeptide enrichment after biotin-tag derivatization, avidin capture, and base treatment. The peaks corresponding to the phosphopeptides shift to $m / z 2166.94$ and 2971.71, respectively, due to the addition of Gly-Cys dipeptide at each of the two sites of phosphorylation. The MALDI-MS spectrum following biotin-avidin affinity enrichment was significantly less complex than that obtained using $\mathrm{TiO}_{2}$ because nonspecific binders such as 1337.76 and 1774.94, which are present in Figure 3b, are absent in Figure 3c.
To demonstrate that the ionization characteristics of the Gly-Cys derivatized peptide were improved, an initial MS/MS experiment was performed on the unmodified phosphorylated precursor peptide after $\mathrm{TiO}_{2}$ enrichment (Figure 4a). In the MS/MS spectrum of the corresponding phosphopeptide, the most abundant and intense peak was that of the doubly charged ion highlighting the difficulty of ionizing and fragmenting these phosphorylated species. When the Gly-Cys modified peptide was fragmented, the triply charged ion was easily identified at the same collision energy settings (Figure $4 \mathrm{~b}$ ). Although the $b$ and $y$ ions dominate the spectrum, the series of $y$ ions are more complete, and better coverage was observed over the high $m / z$ region. The sequence obtained corresponds to EVVGS(GC)AEAGVDAASVSEEFR with an increase in mass of $158.05 \mathrm{Da}$ on the $b_{5}$ to $b_{7}$ ions, which was attributed to the addition of Gly-Cys dipeptide at the site of phosphorylation.

\section{Tryptic Digest; $\alpha$-Casein}

$\alpha$-Casein, a milk-derived protein that possesses multiple phosphorylation sites was also used to confirm our enrichment methodology. The commercially available $\alpha$-casein has two variants with low sequence homology. Carr et al. [30] detected nine phosphorylation sites using precursor ion scanning. However, they were not

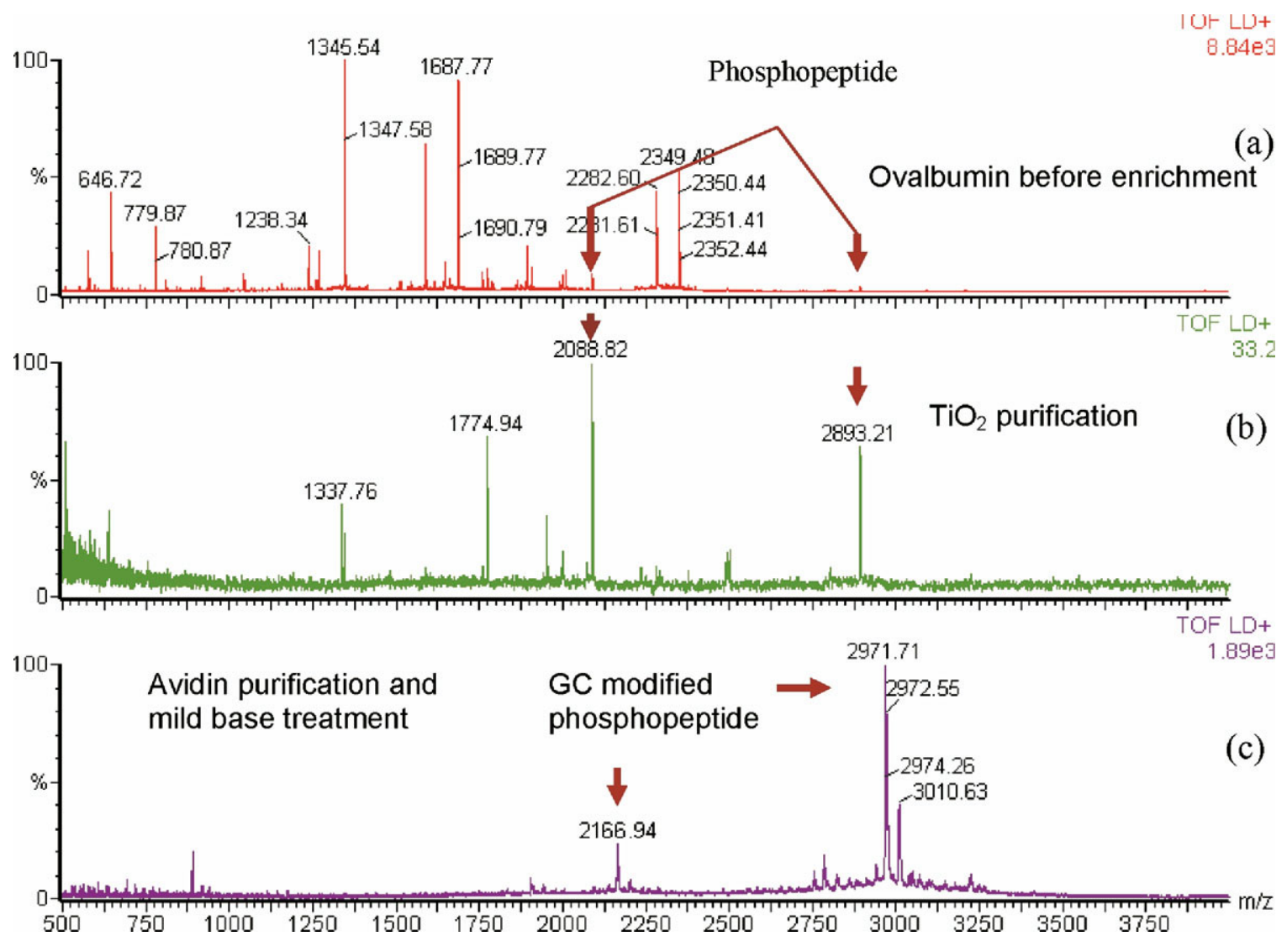

Figure 3. The MALDI-MS spectra of (a) ovalbumin before derivatization, after (b) $\mathrm{TiO}_{2}$ enrichment, (c) avidin capture and base treatment. Two phosphopeptides were enriched which now have Gly-Cys at the sites of phosphorylation. 

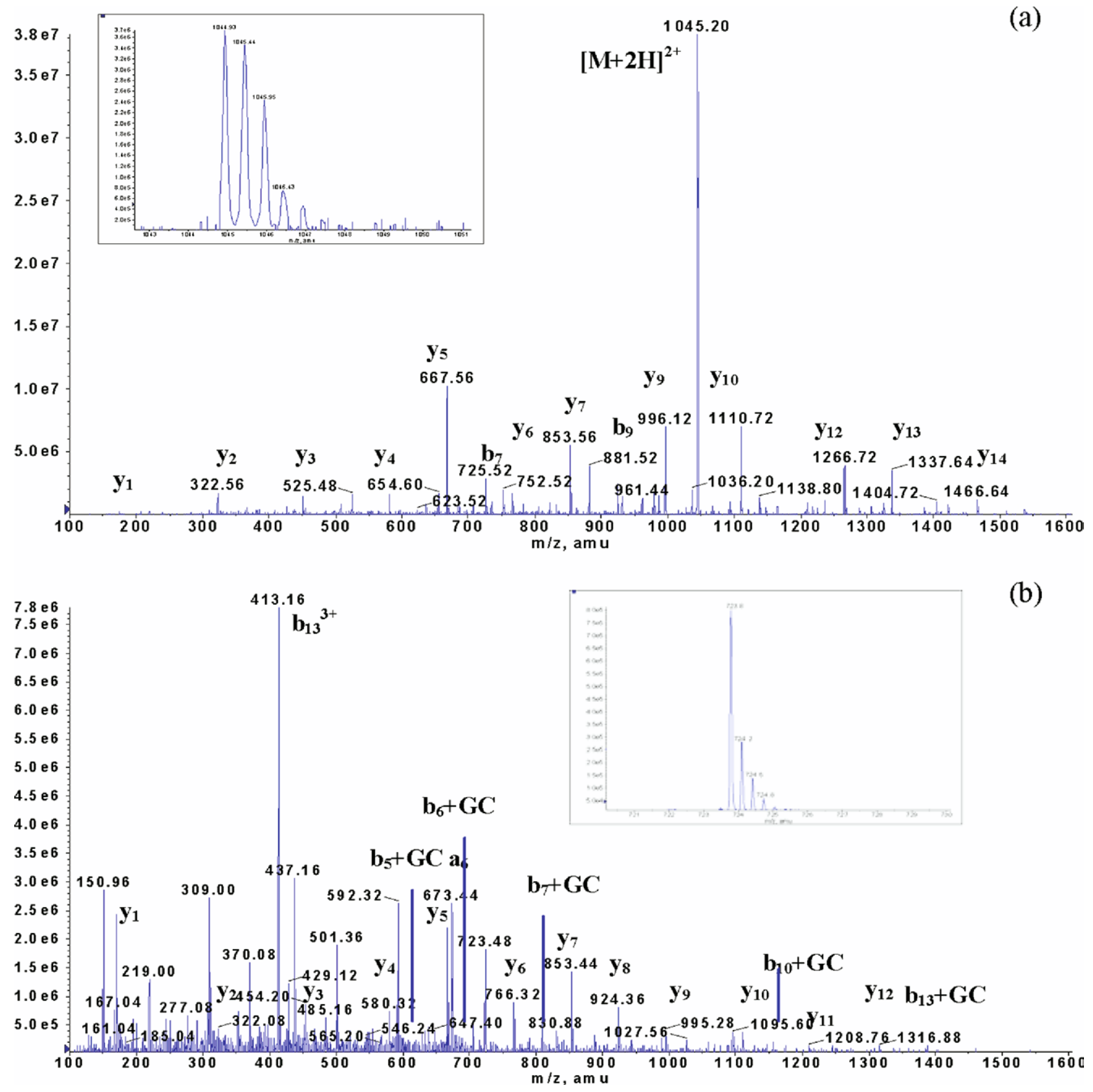

Figure 4. (a) MS/MS spectrum of $m / z 1044.95$ following enrichment with $\mathrm{TiO}_{2}$. The corresponding sequence is EVVGS $\left(\mathrm{PO}_{4}\right)$ AEAGVDAASVSEEFR. Enhanced resolution MS spectrum of doubly charged ion at $\mathrm{m} / \mathrm{z} 1044.95$ (inset). (b) MS/MS spectrum of $\mathrm{m} / \mathrm{z} 723.8$ following avidin enrichment and base treatment to release the Gly-Cys modified peptide. The corresponding sequence is EVVGS (GC)AEAGVDAASVSEEFR. Enhanced resolution MS spectrum of triply charged Gly-Cys modified peptide at $\mathrm{m} / \mathrm{z} 723.8$ (inset).

able to sequence all the phosphopeptides, especially those phosphopeptides with multiple phosphorylation sites, because of the ionization suppression effects described previously. The data obtained correlated with that found by MALDI-MS using the His-tag/Factor Xa enrichment strategy [29]. The MS (inset) and MS/MS (Figure 5a) spectra of one of the GC-derivatized peptides from $\alpha$-casein was identified using a QTRAP 4000 MS instrument. Although the $y$ and $b$ ions dominate the spectrum, the series of $y$ ions are more complete, and better coverage was observed over the high $\mathrm{m} / \mathrm{z}$ region. The fragment obtained corresponds to YKVPQLEIVPNSAEER and the mass of 160.04 Da was added to all fragments from $y_{5}$ to $y_{15}$, which is attributed to the addition of Gly-Cys dipeptide at the site of phosphorylation (Ser12).

To demonstrate that the ionization characteristics of the Gly-Cys derivatized peptide improved, another MS/MS experiment was performed on the unmodified 

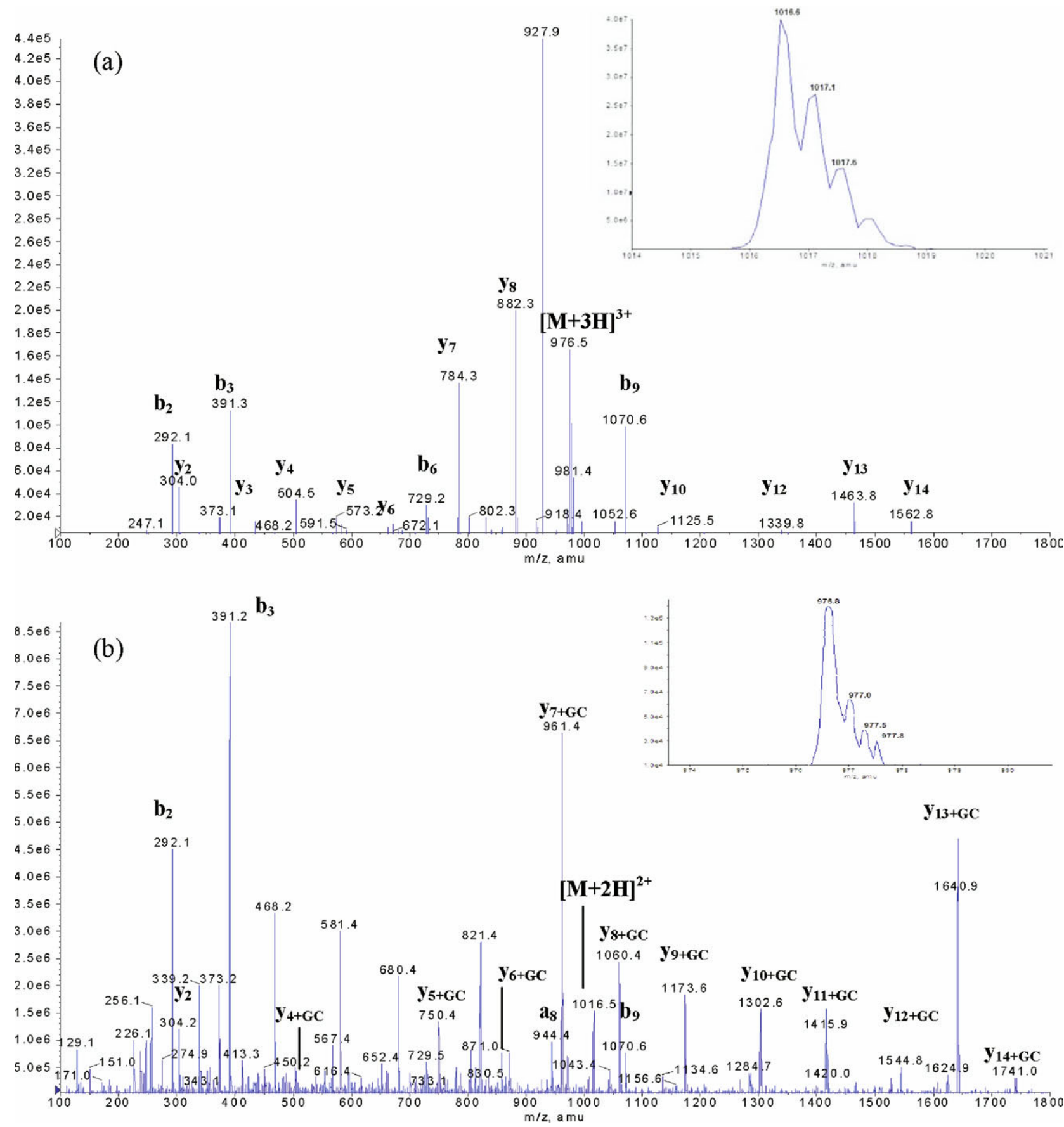

Figure 5. (a) ESI-MS/MS and ESI-MS (inset) spectra of Gly-Cys derivatized $\alpha$-casein phosphopeptide after modification, avidin enrichment, and base treatment $\mathrm{m} / \mathrm{z} 1016.6$ for the $[\mathrm{M}+2 \mathrm{H}]^{2+}$ ion. (b) ESI-MS/MS and ESI-MS spectra (inset) of the precursor phosphopeptide before His-tag derivatization $m / z 976.8$ for the $[\mathrm{M}+3 \mathrm{H}]^{3+}$ ion and corresponds to the YKVPQLEIVPNS(GC)AEER sequence.

phosphorylated precursor peptide using the same collision energy settings (Figure 5b). In the MS/MS spectrum of the corresponding phosphopeptide, the most abundant and intense peak was that of the triply charged ion highlighting the difficulty of ionizing and fragmenting these phosphorylated species. In comparison, the series of $b$ and $y$ ions are far more intense in the case of the peptide modified with Gly-Cys. In agreement with the results, substitution of a negatively- charged phosphate with a protonatable group improved ionization efficiency of the species of interest.

\section{Conclusions}

The combination of chemical derivatization with biotintag and affinity enrichment using an avidin column is a highly efficient approach towards identification of low concentrations of phosphopeptides in complex mix- 
tures. Replacement of the phosphate group with a protonatable Gly-Cys dipeptide following avidin capture and base treatment enhances MS ionization efficiency compared with the phosphorylated precursors, augments CID backbone fragmentation and, as result, facilitates phosphorylation site identification. Furthermore, this procedure adds elements of specificity and selectivity, which aids the elimination of nonspecific sequences that might otherwise compromise the enrichment and subsequent analysis of low concentrations of phosphopeptide. The Gly-Cys modification is also less hydrophilic than the corresponding phosphorylated peptide, thus improving its retention on RP chromatographic media and allowing detection of phosphopeptides that might otherwise have been lost in the solvent front.

Problems associated with the lability of phosphate groups are overcome as a result of the covalent modification with Gly-Cys dipeptide, which remains attached during mass analysis. The method we describe offers some distinct advantages over the previously reported His-tag derivatization approach in that the biotin-tag is smaller and therefore easier to couple, is less complicated to produce, and avoids the need for expensive Factor Xa to release the enriched Gly-Cys modified peptides from the affinity column. The result of this research confirms that this innovative method provides a reliable methodology for phosphopeptide mapping of complex protein mixtures.

\section{References}

1. Aebersold, R.; Watts, J. D.; Morrison, H. D.; Bures, E. Determination of the Site of Tyrosine Phosphorylation at the Low Picomole Level by Automated Solid-Phase Sequence Snalysis. Anal. Biochem. 1991, 199, $51-60$

2. Girault, J. A.; Hemmings, H. C. J.; Williams, K. R.; Nairn, A. C.; Greengard, P. Phosphorylation of DARPP-32, a Dopamine- and cAMPRegulated Phosphoprotein, by Casein Kinase II. J. Biol. Chem. 1989, 264 , $21748-21759$.

3. Annan, R. S.; Carr, S. A. The Essential Role of Mass Spectrometry in Characterizing Protein Structure: Mapping Post-Translational Modifications. J. Protein Chem. 1997, 16, 391-402.

4. Qin, J.; Chait, T. Identification and Characterization of Post-Translational Modifications of Proteins by MALDI Ion Trap Mass Spectrometry. Anal. Chem. 1997, 69, 4002-4009.

5. Yi, Z.; Luo, M.; Mandarino, L. J.; Reyna, S. M.; Carroll, C. A.; Weintraub, S. T. Quantification of Phosphorylation of Insulin Receptor Substrate-1 by HPLC-ESI-MS/MS. J. Am. Soc. Mass Spectrom. 2006, 17, 562-567.

6. Trinidad, C. J.; Specht, G. C.; Thalhammer, A.; Schoepfer, R.; Burlingame, L. A. Comprehensive Identification of Phosphorylation Sites in PostSynaptic Density Preparations. Mol. Cell. Proteom. 2006, 5, 914-922.

7. Lim, K. B.; Kassel, D. B. Phosphopeptides Enrichment Using On-Line Two-Dimensional Strong Cation Exchange Followed by ReversedPhase Liquid Chromatography/Mass Spectrometry. Anal. Biochem. 2006, 354, 213-219.

8. Muszynska, G.; Dobrowolska, G.; Medin, A.; Ekamn, P.; Porath, J. O. Model Studies on Iron (III) Ion Affinity Chromatography II. Interaction of Immobilized Iron (III) Ions with Phosphorylated Amino Acids, Peptides, and Proteins. J. Chromatogr. 1992, 604, 19-28.

9. Sparbier, K.; Koch, S.; Kessler, I.; Wenzel, T.; Kostrzewa, M. Selective Isolation of Glycoproteins and Glycopeptides for MALDI-TOF MS
Detection Supported by Magnetic Particles. J. Biomol. Tech. 2005, 16, 407-413.

10. Dass, C. Principles and Practice of Biological Mass Spectrometry. John Wiley and Sons, Inc: New York, 2001, pp 203-207.

11. Nuwaysir, L. M.; Stults, J. T. Electrospray Ionization Mass Spectrometry of Phosphopeptides Isolated by On-Line Immobilized Metal-Ion Affinity Chromatography. J. Am. Soc. Mass Spectrom. 1993, 4, 662-669.

12. Posewitz, M.; Tempst, P. Immobilized Gallium (III) Affinity Chromatography of Phosphopeptides. Anal. Chem. 1999, 71, 2883-2892.

13. Stensballe, A.; Andersen, S.; Jensen, O. N. Characterization of Phosphoproteins from Electrophoretic Gels by Nanoscale Fe (III) Affinity Chromatography with Off-Line Mass Spectrometry Analysis. Proteomics 2001, 1, 207-222.

14. Brill, M. L.; Salomon, R. A.; Ficarro, B. S.; Mukherji, M.; Stettler-Gill, M.; Peters, C. E. Robust Phosphoproteomic Profiling of Tyrosine Phosphorylation Sites from Human T Cells Using Immobilized Metal Affinity Chromatography and Tandem Mass Spectrometry. Anal. Chem. 2004, 76 , 2763-2772.

15. Dawoud, A. A.; Sarvaiya, H. A.; Lazar, I. M. Microfluidic Platform with Mass Spectrometry Detection for the Analysis of Phosphoproteins. Electrophoresis 2007, 24, 4645-4660.

16. Larsen, M. R.; Thingholm, T. E.; Jensen, O. N.; Roepstorff, P.; Jorgensen, T. J. Highly Selective Enrichment of Phosphorylated Peptides from Peptide Mixtures Using Titanium Dioxide Microcolumns. Mol. Cell. Proteom. 2005, 4, 873-886.

17. Shih-Shin, L.; Makamba, H.; Shang-Yu, H.; Shu-Hui, C. Nanotitanium Dioxide Composites for the Enrichment of Phosphopeptides. J. Chromatogr. A 2006, 1116, 38-45.

18. Kyong, K. H.; Hakansson, K. Selective Zirconium Dioxide-Based Enrichment of Phosphorylated Peptides for Mass Spectrometric Analysis. Anal. Chem. 2006, 78, 1743-1749.

19. Schroeder, M. J.; Shabanowitz, J.; Schwartz, J. C.; Hunt, D. F.; Coon, J. J. A Neutral Loss Activation Method for Improved Phosphopeptide Sequence Analysis by Quadrupole Ion Trap Mass Spectrometry. Anal. Chem. 2004, 76, 3590-3598.

20. Yu, L. R.; Zhu, Z.; Chan, K. C.; Issaq, H. J.; Dimitrov, D. S.; Veenstra, T. D. Improved Titanium Dioxide Enrichment of Phosphopeptides from HeLa Cells and High Confident Phosphopeptide Identification by Cross-Validation of MS/MS and MS/MS/MS Sspectra. J. Proteome Res. 2007, 11, 4150-4162.

21. Wang, Y.; Chen, W.; Wu, J.; Guo, Y.; Xia, X. Highly Efficient and Selective Enrichment of Phosphopeptides Using Porous Anodic Alumina Membrane for MALDI-TOF MS Analysis. J. Am. Soc. Mass Spectrom. 2007, 18, 1387-1395.

22. Li, Y.; Leng, T.; Lin, H.; Deng, C.; Xu, X.; Yao, N.; Yang, P.; Zhang, X. Preparation of $\mathrm{Fe}_{3} \mathrm{O}_{4}$ and $\mathrm{ZrO}_{2}$ Core-Shell Microspheres as Affinity Probes for Selective Enrichment and Direct Determination of Phosphopeptides Using Matrix-Assisted Laser Desorption Ionization Mass Spectrometry. J. Proteome Res. 2007, 11, 4498-4510.

23. Xu, X.; Deng, C.; Gao, M.; Yu, W.; Yang, P.; Zhang, X. Synthesis of Magnetic Microspheres with Immobilized Metal Ions for Enrichment and Direct Determination of Phosphopeptides by Matrix-Assisted Laser Desorption Ionization Mass Spectrometry. Adv. Mater. 2006, 18, 3289 3293.

24. Amoresano, A.; Monti, G.; Cirulli, C.; Marino, G. Selective Detection and Identification of Phosphopeptides by Dansyl MS/MS/MS Fragmentation. Rapid Commun. Mass Spectrom. 2006, 20, 1400-1404.

25. Klemm, C.; Schröder, S.; Glückmann, M.; Beyermann, M.; Krause, E. Derivatization of Phosphorylated Peptides with S- and N-Nucleophiles for Enhanced Ionization Efficiency in Matrix-Assisted Laser Desorption/ Ionization Mass Spectrometry. Rapid Commun. Mass Spectrom. 2004, 18, 2697-2705.

26. Knight, Z. A.; Schilling, B.; Row, R. H.; Kenski, D. M.; Gibson, B. W.; Shokat, K. M. Phosphospecific Proteolysis for Mapping Sites of Protein Phosphorylation. Nat. Biotechnol. 2003, 21, 1047-1054

27. Brittain, S. M.; Ficarro, S. B.; Brock, A.; Peters, E. C. Enrichment and Analysis of Peptide Subsets Using Fluorous Affinity Tags and Mass Spectrometry. Nat. Biotechnol. 2005, 23, 463-468.

28. Adamczyk, M.; Gebler, J. C.; Wu, J. Selective Analysis of Phosphopeptides Within a Protein Mixture by Chemical Modification, Reversible Biotinylation, and Mass Spectrometry. Rapid Commun. Mass Spectrom. 2001, 15, 1481-1488.

29. Jalili, P. R.; Sharma, D.; Ball, H. L. Enhancement of Ionization Efficiency and Selective Enrichment of Phosphorylated Peptides from Complex Protein Mixtures Using a Reversible Polyhistidine Tag. J. Am. Soc. Mass Spectrom. 2007, 18, 1007-1017.

30. Carr, A. S.; Huddleston, J. M.; Annan, S. R. Selective Detection and Sequencing of Phosphopeptides at the Femtomole Level by Mass Spectrometry. Anal. Biochem. 1996, 239, 180-192. 